\title{
Esthetic Restoration of Enamel Hypoplasia: A Case Report Maida Fitri ${ }^{1,3^{*}}$ Kamizar, Kamizar ${ }^{2}$
}

\author{
${ }^{1}$ Postgraduate of Conservative Dentistry Program, Faculty of Dentistry, Universitas Indonesia, Jakarta, Indonesia \\ ${ }^{2}$ Lecturer of Department Conservative dentistry, Faculty of Dentistry, Indonesia University, Jakarta, Indonesia \\ ${ }^{3}$ Lecturer of Department Conservative dentistry, Faculty of Dentistry, Universitas Syiah Kuala, Aceh, Indonesia \\ * Corresponding author. Email: maidadrg06@unsyiah.ac.id
}

\begin{abstract}
Introduction: Enamel hypoplasia is an abnormality condition of enamel that occurs because of disturbance during the process of amelogenesis. Clinically, enamel hypoplasia can be thin enamel or absence of enamel. It can appear white, yellow, or brownish color with a rough or pitted surface, bilaterally, often in the middle third area of the crown, size (depth and extent) of the defect that is directly related to severity. Treatment for enamel hypoplasia disorders depends on their severity. Objective: To describe the condition and management of enamel hypoplasia case that causes esthetic disorders. Case Report: A 17-year-old man came to the Conservative Dentistry Department of RSKGM FKG UI with the chief complaint of the brown striped color of anterior teeth in the upper and lower jaw. The abnormalities on these teeth have occurred since his childhood, and now he starts to feel unconfident. From the clinical examination, maxillary and mandibular anterior and premolar teeth showed white-brown lesions on the labial surface. The diagnosis is enamel hypoplasia type III and will be restored with a composite resin restoration as the treatment plan. Conclusion: Enamel hypoplasia can be treated by direct restoration with composite resin to restore its colors and to look similar to natural teeth. No discoloration was found after two months and patients feel satisfied.
\end{abstract}

\section{Keywords: Enamel hypoplasia, Composite resin, Esthetic, Direct restoration, Discoloration}

\section{INTRODUCTION}

The aesthetic need to get a perfect smile is increasing from year to year. It relates to the shape, position, color, and alignment of teeth well. ${ }^{1}$

The color and shape of the teeth are a condition caused by many factors such as lighting conditions, translucency, opacity, light transmission, and human perception. Tooth color is determined by the translucency of the dentin and enamel. The tubules are the main cause of light transmission occurring in dentin and enamel which hydroxyapatite crystals are contributing significantly to the light transmission. ${ }^{2}$

Enamel is an outer structure and the most important part of the teeth, both functionally and aesthetically. The formation of enamel is a unique biomineralization process involving deposition and degradation of matrix proteins that are highly organized. Ameloblasts are cells derived from ectodermal which responsible for the growth and development of enamel. ${ }^{3}$

These cells secrete enamel protein which is necessary for mineralization and maturation. Enamel formation characterized by the inductive phase, secretion, and maturation. During the inductive phase, enamel epithelium begins to differentiate. Later stages of the secretion, differentiated of ameloblast enamel release proteins which contribute to the enamel matrix. Recently at the stage of maturation, ameloblast absorb water and organic matrix, resulting in the crystal deposition. ${ }^{3}$

The growth process of the teeth can be impaired resulting in the occurrence of some abnormalities. One of tooth disorders is abnormality of the structure of the tooth enamel which can occur when a disturbance during the formation of the matrix enamel, so it looks like defects in the enamel, known as hypoplastic enamel. ${ }^{4}$

Enamel hypoplasia is a disorder in the enamel due to interruption during the process of amelogenesis. Amelogenesis occurs in two phases, namely the secretion of the matrix by ameloblast and maturation process. Ameloblast activity may be hampered by some environmental factors such as systemic physiological stress, thereby reducing the number of enamels matrix secretion. At the end of the secretion phase, thinner enamel will appear in the areas involved. ${ }^{3}$

Clinically, enamel hypoplasia formed a thin enamel and their enamel can be shaped pit or groove, horizontally or vertically on the tooth surface. This disorder usually occurs bilaterally, both the labial and lingual surfaces. Locations affected area usually at the middle third of the crown, followed by the cervical area and incisal/occlusal. The size (depth and breadth) defect that occurs directly related to the level of severity. ${ }^{3}$

Factors that influence the occurrence of enamel hypoplasia are hereditary factors and environmental 
factors. In hereditary factors, enamel hypoplasia was known as amelogenesis imperfecta, while the causes that derived from environmental factors which influence the occurrence of this disorder are trauma, malnutrition (lack of vitamin A, C, and D) and infectious disease (congenital syphilis, exanthematous disease). ${ }^{3,5}$

The frequency of disturbance during the development of enamel in the permanent dentition showed a high rate. Robles et al mention that there are about $52 \%$ of enamel defects occur due to tooth development permanent disorder.9 Sandhu et al say that defects in permanent teeth growth occurs due to interference between $12 \%-69 \% .^{6}$

Treatment of enamel hypoplasia varies depending on the severity of the lesion. Some conventional approach are micro-abrasion enamel, conservative restoration and also dental bleaching. ${ }^{4}$ This case report will be discussed in the treatment of teeth with enamel hypoplasia using composite resin.

\section{CASE REPORT}

Male patient, aged 17, was referred with her mother to the conservative dentistry clinic RSKGM Faculty of Dentistry, Indonesia University. Patients complain of his front teeth brown colored stripes on the upper jaw and lower jaw. Abnormalities in the teeth had been there since childhood, but now patients begin to feel disturbed by these disorders. so that the patient wants all the teeth abnormalities can be restored as normal teeth shape.

Based on clinical examination, was found enamel defect at 11, 12, 13, 14, 21, 22, 23, 24, 33, 43, 34, and 44. The diagnosis is enamel hypoplasia and the treatment plan is direct composite resin restorations.

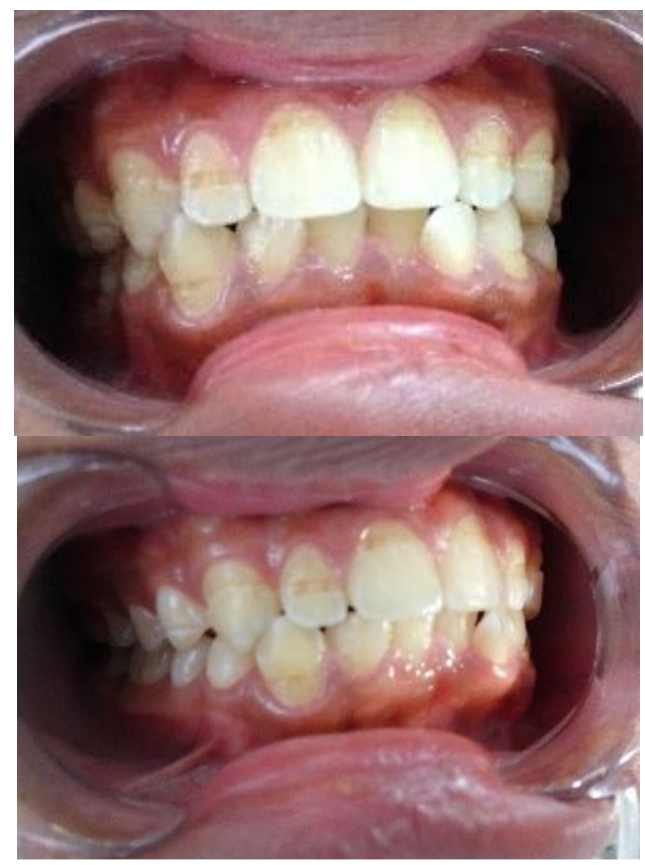

Figure 1. Initial photo from clinical examiation, above: front view (left), looks right (right)

\section{CASE MANAGEMENT}

The first visit taking historical and complete clinical examination and continued with restorations in the teeth of the upper jaw, namely 11, 12, 13, 14, 21, 22, 23 and 24. Shade selection should be determined before isolating teeth. The teeth was isolated with a rubber dam (Nictone), then brushed on the labial surface, so that debris and plaque disappear. After that, made a minimal preparation, then the teeth was etched for 15 seconds, washed, applied bonding agent (3M ESPE Adper), and then curing. Restoration used microhybrid composite resin (3M ESPE Z250 Universal) shade A3,5 for lesions in cervical area, and shade A3 for lesions in incisal area, then added translucent color. The application tools restoration instrument (LMarte), used to applied the material and then cured with a unit light-emitting diode (LED) for 20 seconds. Polished the composite resin used polishing tools (soflex $3 \mathrm{M}$ ).

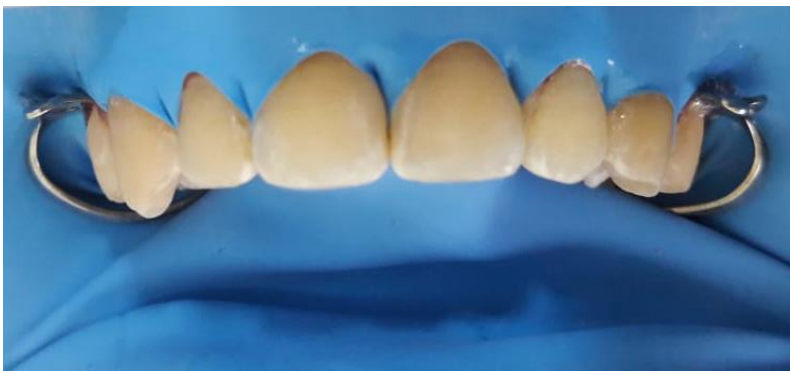

Figure 2. After the restoration of RK to the teeth of the upper jaw

The next visit, restore the mandibular teeth with the same method. Before teeth preparation, applied the rubber dam (Nictone) then brushed the labial surface of the tooth so that debris and plaque disappear. Then, minimal preparation was done. After that, the tooth was etched, cleaned, and applied bonding agent (3M ESPE Adper) in the cavities. Restoration is done with microhybrid composite resin (3M ESPE Z250 Universal) shade A3,5 for lesions in the cervical area and shade A3 for lesions in the incisal area, then added translucent color, cured with the light-emitting diode (LED) unit for 20 seconds. Polished the composite resin using soflex to made smooth surface.

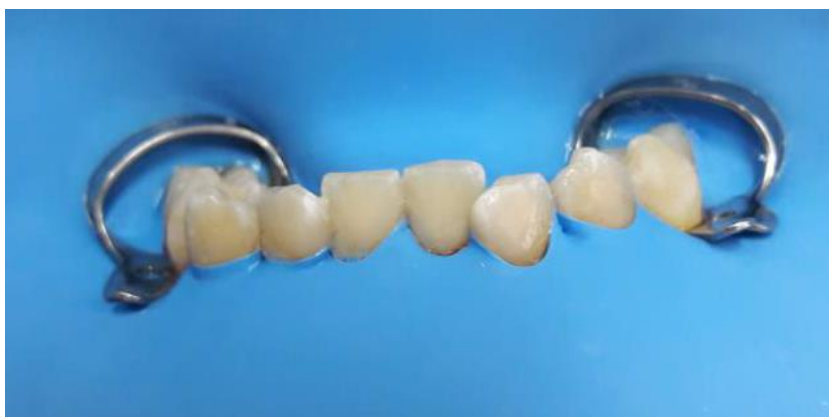

Figure 3. After the restoration of Composite Resin in the teeth of the lower jaw 
Followed up at 2 months after the restoration and the results showed no complaints from patients. Based on clinical examination restoration complete with a smooth composite surface and there is no discoloration of the restorative material.

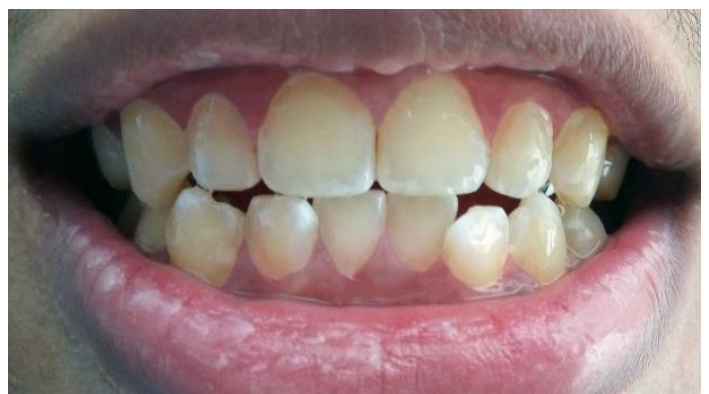

Figure 4. Evaluation after 2 months

\section{DISCUSSION}

This case report describes the brownish enamel lesions on the anterior until premolar teeth of the upper and lower jaw. Symmetrical left and right lesion indicated that the abnormality originates from the stage of growth and development, followed by loosing enamel in certain parts.

Hypoplasia categorized into several types by Silbermann et al: ${ }^{6,7}$

Hypoplasia Type I: Enamel discoloration caused hypoplasia.

Hypoplasia Type II: Abnormal coalescence caused hypoplasia

Hypoplasia Type III: Some parts missing enamels caused hypoplasia

Hypoplasia Type IV: A combination of three types of hypoplasia (hypoplasia enamel circular).

Classifying hypoplasia can also use a specific index, which is 0: no hypoplasia; I: hypoplasia with aplastic defects in the enamel; II: hypoplasia with aplastic defects in the enamel and dentin; and III: hypoplasia with an irregular tooth crown, and discoloration that is a: no discoloration; b: white opacities in enamel; c: yellowbrownish enamel discoloration. ${ }^{4}$ So this disorder can be summarized as enamel hypoplasia type III. Because of enamel lesions are mild and there is only one-third section of the cervical or cervical middle third or onethird, so the proper care is by performing direct composite resin restorations. This treatment is appropriate because in line with the principle of minimal invasive and has the aesthetic function.

Composite resins are tooth-colored restorations that has an aesthetic value. The content of composite resin is a polymer matrix organic, inorganic filler, coupling agent, initiator, and activator system, and the pigment that gives shades on it. ${ }^{8,9}$ Filler size of this material affects the optical and mechanical properties of the composite. The larger particles make it ease to light deflected to be opaque or translucent, so that it has a low depth of cure, but it is also more coarse texture, make it easier to create stain and plaque retention. Meanwhile, the smaller the particle size, then the lighter it is. It is also not easily deflected and creates translucency, so that the depth of cure is also increasing. Besides the smaller particles also create a smooth surface texture, it also make composite looks more esthetic. ${ }^{8}$

Composite resin restorations which used in this case is microhybrid composite. Hybrid composites are composites typed with different filler size, so the smaller size of the filler can fill the void between the larger filler. The composite resin which used containing (3M ESPE Z250 Universal) UDMA resin matrix can keep the color stability of composite resin for low viscosity and low water absorption. Polymerization process using lightemitting diode (LED) that show color changes slightly on the composite resin. The content photoinitiator (camphoroquinone) with the right composition during the polymerization of the composite resin causing less color change after polymerized, so that still maintain the color like before polymerization. ${ }^{10,11}$

To get the criteria obtained more optimal aesthetic, composite color selection strategy was conducted by taking a small number of restorative materials without etching and bonding, placed on the teeth near the area to be restored then the in-curing materials. This step can improve the accuracy in the determination of the color. If the selected color was appropriate, the material removed from the tooth surface by using explorer. Shade selection should be determined before isolating teeth so that color variations that can occure as a result of drying and dehydration of teeth are avoided. ${ }^{1}$

Etching procedure applied here is a total-etch system, which can eliminate the smear layer across the surface of the teeth after the preparing procedure, thereby increasing the surface energy of the enamel. Etching gel was applied for 15 seconds to make effectively shape of 30 micrometers of microporosity on the enamel prism. The dentin tubules layer will be open so that there is a hybrid layer to produce a complete bond between the tooth and the bonding agent. The washing procedure is done to clean the etching of the tooth surface, and dampness in the teeth to make the collagen in the dentinal tubules did not collapse. The bonding process is done to form resin tags to penetrate spaces around the prism of enamel and into tubular dentin. ${ }^{12}$

LMarte instrument is an instrument to apply resin composite, made by super steel of high quality with a thin design and a smooth surface, flexible, easy-tohandle and good precision. It makes this instrument suitable for the application of composite resins both in anterior and posterior regions. ${ }^{13}$

\section{CONCLUSION}

In this case, the tooth with type III enamel hypoplasia disorder was treated using a micro-hybrid composite resin direct restorations. Accurate shade selection is best attained by applying and curing a small 
amount of the composite restorative material in the area of the tooth that may need restoration. In two months of evaluation, the tooth color has not changed and the patient is satisfied.

\section{ACKNOWLEDGMENT}

The authors would like to thank RSKGM and Department of Conservative Dentistry, Faculty of Dentistry, Universitas Indonesia for supporting this case report.

\section{CONFLICT OF INTEREST}

The authors do not declare any conflicts of interest.

\section{REFERENCES}

1. Heymann HO, Swift EJ, \& Ritter AV. Sturdevant's Art and Science of Operative Dentistry. $10^{\text {th }}$ ed. Elsevier Mosby: Philadelphia. 2013.

2. Daniel Awad, et al. Translucency of Esthetic Dental Restorative CAD/CAM Materials and Composite Resins with Respect to Thickness and Surface Roughnes. J Prosthet Dent. 2015.

3. Simalcsik RD, Simalcsik A, \& Groza VM. Dental Enamel Hypolplasia. Investigations on the Bones Exhumed from the Medieval Necropole of Lozova (Republic of Moldova), $\mathrm{XIV}^{\text {th }}-\mathrm{XV}^{\text {th }}$ Centuries. Memoirs of the Scientific Sections of the Romanian Academy. 2014.

4. Gupta SP, Shetty PP, \& Reddy K. Enamel Hipoplasia: A Case Report. Journal of Advanced Oral Research. 2014. 5:1.

5. Garg TM \& Krishan S. Enamel Hypoplasia and Rehabilitation of Esthetics: A Case Report. Arch of Dent and Med Res. 2016. 2(6): 24-27.

6. Sandhu M, Gulia S, \& Nagpal M. Circular Enamel Hipoplasia: A Rare Enamel Developmental Disturbance in Permanent Teeth. Journal of Clinical and Diagnostic Research. 2014. 8(8): ZD39-ZD40.

7. Vanishree HS, Tegginamani AS. Enamel Hypoplasia: A Concise Review of Its Factors \& Pathogenesis. American Journal of Oral Medicine and Radiology. 2016. 3(1): 48-51.

8. Sakaguchi RL \& Powers JM. Craig's Restorative Dental Materials. $13^{\text {th }}$ ed. Elsevier Mosby: Philadelphia. 2012.

9. Mount, G.J. Hume, W.R. Preservation and Restoration of Tooth Structure. $3^{\text {rd }}$ ed. Knowledge Books: Queensland. 2016.

10. Yew HZ, Qamaruz Zaman J. Colour Stability of Composite Resins: A General Overview. Malaysian Dental Journal. 2012. Volume 34 (Issue 2).

11. Nandhini GA \& Jayalakshmi, S. Factors that Influence the Color Stability of Composite Restorations. International Journal of Orofacial Biology. 2017. Volume 1 (Issue 1).
12. Malik S, Bal C, \& Singh R. Tensile Bond Strength of Total Etch and Self Etch Adhesive on Moist and Dry Dentin In-Vivo Study. Indian Journal of Comprehensive Dental Care. 2014. 4: 422-427.

13. LM-Dental. Lm-Arte ${ }^{\mathrm{TM}}$. Aesthetic LayeringSucceed with LM-Arte. Available on: https://www.lm-dental.com/products/handinstrumentation/restoration/lm-arte/ 